\title{
TOTALLY REAL PSEUDO-UMBILICAL SUBMANIFOLDS OF A QUATERNION SPACE FORM
}

\author{
by HUAFEI SUN†
}

(Received 12 June, 1996)

1. Introduction. Let $M(c)$ denote a $4 n$-dimensional quaternion space form of quaternion sectional curvature $c$, and let $P(H)$ denote the $4 n$-dimensional quaternion projective space of constant quaternion sectional curvature 4 . Let $N$ be an $n$-dimensional Riemannian manifold isometrically immersed in $M(c)$. We call $N$ a totally real submanifold of $M(c)$ if each tangent 2-plane of $N$ is mapped into a totally real plane in $M(c)$. B. Y. Chen and C. S. Houh proved in [1].

Theorem A. Let $M$ be an n-dimensional compact totally real minimal submanifold of the quaternion projective space $P(H)$. If

$$
S \leq \frac{3 n(n+1)}{(6 n-1)},
$$

then $N$ is totally geodesic. Here $S$ is the square of the length of the second fundamental form of $N$.

Let $h$ be the second fundamental form of the immersion, and $\xi$ the mean curvature vector. Let $\langle\cdot, \cdot\rangle$ denote the scalar product of $M(c)$. If there exists a function $\lambda$ on $N$ such that

$$
\langle h(X, Y), \xi\rangle=\lambda\langle X, Y\rangle
$$

for any tangent vector $X, Y$ on $N$, then $N$ is called a pseudo-umbilical submanifold of $M(c)$. It is clear that $\lambda \geq 0$. If the mean curvature vector $\xi=0$ identically, then $N$ is called a minimal submanifold of $M(c)$. Every minimal submanifold of $M(c)$ is itself a pseudo-umbilical submanifold of $M(c)$. In this paper, we consider the case when $N$ is pseudo-umbilical and extend Theorem A. Our main results are

THEOREM 1. Let $N$ be an n-dimensional compact totally real pseudo-umbilical submanifold of $M(c)$. Then

$$
\int_{N}\left\{6 S^{2}-\left[(n+1) c+16 n H^{2}\right] S+4 n^{2} H^{2} c+10 n^{2} H^{4}\right\} d N \geq 0
$$

where $H$ and $d N$ denote the mean curvature of $N$ and the volume element of $N$ respectively.

THEOREM 2. Let $N$ be an n-dimensional compact totally real submanifold of $M(c)$. If

$$
6 S^{2}-\left((n+1) c+16 n H^{2}\right) S+4 n^{2} H^{2} c+10 n^{2} H^{4}-4 n H \Delta H \leq 0,
$$

then the second fundamental form of $N$ is parallel. In particular, if the equality of (1.1) holds, then either $N$ is totally geodesic or $N$ is flat.

When $H \equiv 0$, i.e. $N$ is minimal, from Theorem 1 we may get (cf. [4]).

$\dagger I$ would like to thank Prof. K. Ogiue for his advice and encouragement and to thank also the referee for valuable suggestions. 
COROLlaRY. Let $N$ be an n-dimensional compact totally real minimal submanifold of $P(H)$. If

$$
S \leq \frac{2}{3}(n+1),
$$

then $N$ is totally geodesic or $S=\frac{2}{3}(n+1)$.

2. Local formulas. We use the same notation and terminologies as in [1] unless otherwise stated. Let $M(c)$ denote a $4 n$-dimensional quaternion space form of quaternion sectional curvature $c$, and let $N$ be an $n$-dimensional totally real submanifold of $M(c)$. We choose a local field of orthonormal frames,

$$
\begin{gathered}
e_{1}, \ldots, e_{n}, \quad e_{I(1)}=I e_{1}, \ldots, e_{I(n)}=I e_{n}, \\
e_{J(1)}=J e_{1}, \ldots, e_{J(n)}=J e_{n}, \quad e_{k(1)}=K e_{1}, \ldots, e_{k(n)}=K e_{n},
\end{gathered}
$$

in such a way that when restricted to $N, e_{1}, \ldots, e_{n}$ are tangent to $N$. Here $I, J, K$ are the almost Hermitan structures and satisfy

$$
I J=-J I=K, J K=-K J=I, K I=-I K=J, I^{2}=J^{2}=K^{2}=-1 .
$$

We shall use the following convention on the range of indices:

$$
\begin{gathered}
A, B, \ldots=1, \ldots, n, I(1), \ldots, I(n), J(1), \ldots, J(n), K(1), \ldots, K(n), \\
\alpha, \beta, \ldots=I(1), \ldots, I(n), J(1), \ldots, J(n), K(1), \ldots, K(n), \\
i, j, \ldots=1, \ldots, n, \quad \phi=I, J, K .
\end{gathered}
$$

Let $\left\{\omega_{A}\right\}$ be the dual frame field. Then the structure equations of $M(c)$ are given by

$$
\begin{aligned}
d \omega_{A} & =-\sum_{B} \omega_{A B} \wedge \omega_{B}, \omega_{A B}+\omega_{B A}=0, \\
d \omega_{A B} & =-\sum_{C} \omega_{A C} \wedge \omega_{C B}+\frac{1}{2} \sum_{C D} K_{A B C D} \omega_{C} \wedge \omega_{D}, \\
K_{A B C D}= & \frac{c}{4}\left(\delta_{A C} \delta_{B D}-\delta_{A D} \delta_{B C}+I_{A C} I_{B D}-I_{A D} I_{B C}+2 I_{A B} I_{C D}+J_{A C} J_{B D}\right. \\
& \left.-J_{A D} J_{B C}+2 J_{A B} J_{C D}+K_{A C} K_{B D}-K_{A D} K_{B C}+2 K_{A B} K_{C D}\right) .
\end{aligned}
$$

Restricting these forms to $N$, we get the following structure equations of the immersion:

$$
\begin{aligned}
\omega_{\alpha} & =0, \quad \omega_{\alpha i}=\sum_{j} h_{i j}^{\alpha} \omega_{j}, \quad h_{i j}^{\alpha}=h_{j i}^{\alpha}, \quad h_{j k}^{\phi(i)}=h_{i k}^{\phi(j)}=h_{i j}^{\phi(k)}, \\
d \omega_{i j} & =-\sum_{k} \omega_{i k} \wedge \omega_{k j}+\frac{1}{2} \sum_{k l} R_{i j k l} \omega_{k} \wedge \omega_{l}, \\
R_{i j k l} & =K_{i j k l}+\sum_{\alpha}\left(h_{i k}^{\alpha} h_{j l}^{\alpha}-h_{i l}^{\alpha} h_{j k}^{\alpha}\right), \\
d \omega_{\alpha \beta} & =-\sum_{\gamma} \omega_{\alpha \gamma} \wedge \omega_{\gamma \beta}+\frac{1}{2} \sum_{i j} R_{\alpha \beta i j} \omega_{i} \wedge \omega_{j}, \\
R_{\alpha \beta i j} & =K_{\alpha \beta i j}+\sum_{k}\left(h_{i k}^{\alpha} h_{k j}^{\beta}-h_{i k}^{\beta} h_{k j}^{\alpha}\right) .
\end{aligned}
$$


We call $h=\sum_{i j \alpha} h_{i j}^{\alpha} \omega_{i} \omega_{j} e_{\alpha}$ the second fundamental form of the immersed manifold $N$. We denote by $S=\sum_{i j \alpha}\left(h_{i j}^{\alpha}\right)^{2}$ the square of the length of $h . \xi=\frac{1}{n} \sum_{\alpha} \operatorname{tr} H_{\alpha} e_{\alpha r}$ and $H=$ $\frac{1}{n} \sqrt{\sum_{\alpha}\left(\operatorname{tr} H_{\alpha}\right)^{2}}$ denote the mean curvature vector and the mean curvature of $N$, respectively. Here $\operatorname{tr}$ is the trace of the matrix $H_{\alpha}=\left(h_{i j}^{\alpha}\right)$. Now, let $e_{k(n)}$ be parallel to $\xi$. Then we have

$$
\operatorname{tr} H_{k(n)}=n H, \quad \operatorname{tr} H_{\alpha}=0, \alpha \neq k(n) .
$$

We define $h_{i j k}^{\alpha}$ and $h_{i j k l}^{\alpha}$ by

$$
\sum_{k} h_{i j k}^{\alpha} \omega_{k}=d h_{i j}^{\alpha}-\sum_{l} h_{i l}^{\alpha} \omega_{l j}-\sum_{l} h_{l j}^{\alpha} \omega_{l i}+\sum_{\beta} h_{i j}^{\beta} \omega_{\alpha \beta}
$$

and

$$
\sum_{l} h_{i j k l}^{\alpha} \omega_{l}=d h_{i j k}^{\alpha}-\sum_{l} h_{l j k}^{\alpha} \omega_{l i}-\sum_{l} h_{i k k}^{\alpha} \omega_{l j}-\sum_{l} h_{i j l}^{\alpha} \omega_{l k}+\sum_{\beta} h_{i j k}^{\beta} \omega_{\alpha \beta}
$$

respectively. Where

$$
h_{i j k}^{\alpha}=h_{i k j}^{\alpha}
$$

and

$$
h_{i j k l}^{\alpha}-h_{i j k l}^{\alpha}=\sum_{m} h_{i m}^{\alpha} R_{m j k l}+\sum_{m} h_{m j}^{\alpha} R_{m i k l}-\sum_{\beta} h_{i j}^{\beta} R_{\alpha \beta k l}
$$

The Laplacian $h_{i j}^{\alpha}$ of the second fundamental form $h_{i j}^{\alpha}$ is defined by $\Delta h_{i j}^{\alpha}=\sum_{k} h_{i j k k}^{\alpha}$. By a direct calculation we have (cf. $[1,2,3]$ )

$$
\begin{aligned}
\frac{1}{2} \Delta S & =\sum_{i j k \alpha}\left(h_{i j k}^{\alpha}\right)^{2}+\sum_{i j \alpha \alpha} h_{i j}^{\alpha} \Delta h_{i j}^{\alpha} \\
& =\sum_{i j k \alpha}\left(h_{i j k}^{\alpha}\right)^{2}+\sum_{i j k \alpha} h_{i j}^{\alpha} h_{k k j j}^{\alpha}+\sum_{i j k l \alpha} h_{i j}^{\alpha} h_{l k}^{\alpha} R_{l i j k}+\sum_{i j k l \alpha} h_{i j}^{\alpha} h_{i i}^{\alpha} R_{l k j k}+\sum_{i j k \alpha \beta} h_{i j}^{\alpha} h_{i k}^{\beta} R_{\beta \alpha j k} .
\end{aligned}
$$

3. Proofs of Theorems. From (*) and (2.4) we get $\sum_{\alpha} \operatorname{tr} H_{\alpha} h_{i j}^{\alpha}=n \lambda \delta_{i j}, H^{2}=\lambda$ and

$$
h_{i j}^{k(n)}=H \delta_{i j}
$$

Using (3.1) we have

$$
\sum_{i j k \alpha} h_{i j}^{\alpha} h_{k k i j}^{\alpha}=n H \Delta H .
$$


Using (2.1)-(2.4) and (3.1), we derive (cf. $[1,2,3])$

$$
\begin{aligned}
\sum_{i j k l \alpha} & h_{i j}^{\alpha} h_{k l}^{\alpha} R_{l i j k}+\sum_{i j k l \alpha} h_{j i}^{\alpha} h_{i l}^{\alpha} R_{l k j k}+\sum_{i j k \alpha \beta} h_{i j}^{\alpha} h_{l k}^{\beta} R_{\beta \alpha j k} \\
& =\frac{c}{4}(n+1) S-n^{2} H^{2} c+\sum_{i j k l \alpha \beta} h_{k k}^{\alpha} h_{i j}^{\alpha} h_{j i}^{\beta} h_{l i}^{\beta}+\sum_{\alpha \beta} \operatorname{tr}\left(H_{\alpha} H_{\beta}-H_{\beta} H_{\alpha}\right)^{2}-\sum_{\alpha \beta}\left(\operatorname{tr} H_{\alpha} H_{\beta}\right)^{2} \\
& =\frac{c}{4}(n+1) S-n^{2} H^{2} c+n H^{2} S+\sum_{\alpha \beta} \operatorname{tr}\left(H_{\alpha} H_{\beta}-H_{\beta} H_{\alpha}\right)^{2}-\sum_{\alpha \beta}\left(\operatorname{tr} H_{\alpha} H_{\beta}\right)^{2} .
\end{aligned}
$$

Substituting (3.2) and (3.3) into (2.6), we obtain

$$
\frac{1}{2} \Delta S=\sum_{i j k \alpha}\left(h_{i j k}^{\alpha}\right)^{2}+n H \Delta H+\frac{c}{4}(n+1) S-n^{2} H^{2} c+\sum_{\alpha \beta} \operatorname{tr}\left(H_{\alpha} H_{\beta}-H_{\beta} H_{\alpha}\right)^{2}-\sum_{\alpha \beta}\left(\operatorname{tr} H_{\alpha} H_{\beta}\right)^{2} .
$$

In order to prove our Theorems, we need the following Lemmas.

Lemma 1 [4]. Let $H_{i}, i \geq 2$ be symmetric $n \times n$-matrices, $S_{i}=\operatorname{tr} H_{i}^{2}, S=\sum_{i} S_{i}$. Then

$$
\sum_{i j} \operatorname{tr}\left(H_{i} H_{j}-H_{j} H_{i}\right)^{2}-\sum_{i j}\left(\operatorname{tr} H_{i} H_{j}\right)^{2} \geq-\frac{3}{2} S^{2},
$$

and the equality holds if and only if all $H_{i}=0$ or there exist two of $H_{i}$ different from zero. Moreover, if $H_{1} \neq 0, H_{2} \neq 0, H_{i}=0, i \neq 1,2$, then $S_{1}=S_{2}$ and there exists an orthogonal $(n \times n)$-matrix $T$ such that

$$
T H_{1}{ }^{\prime} T=\left(\begin{array}{ccc}
a & 0 & 0 \\
0 & -a & \\
0 & & 0
\end{array}\right), \quad T H_{2}{ }^{T} T=\left(\begin{array}{ccc}
0 & \alpha & 0 \\
a & 0 & \\
0 & & 0
\end{array}\right),
$$

where $a=\sqrt{\frac{S_{1}}{2}}$.

\section{LEMMA 2.}

$$
\sum_{\alpha \beta} \operatorname{tr}\left(H_{\alpha} H_{\beta}-H_{\beta} H_{\alpha}\right)^{2}-\sum_{\alpha \beta}\left(\operatorname{tr} H_{\alpha} H_{\beta}\right)^{2} \geq \frac{3}{2} S^{2}+3 n H^{2} S-\frac{5}{2} n^{2} H^{4} .
$$

In fact, using (2.4), (3.1) and noting that $\alpha$ runs up to $3 n>2$, we have

$$
\begin{aligned}
\sum_{\alpha \beta} \operatorname{tr}\left(H_{\alpha} H_{\beta}-H_{\beta} H_{\alpha}\right)^{2}-\sum_{\alpha \beta}\left(\operatorname{tr} H_{\alpha} H_{\beta}\right)^{2}= & \sum_{\alpha \beta \neq k(n)} \operatorname{tr}\left(H_{\alpha} H_{\beta}-H_{\beta} H_{\alpha}\right)^{2} \\
& -\sum_{\alpha \beta \neq k(n)}\left(\operatorname{tr} H_{\alpha} H_{\beta}\right)^{2}-\left(\operatorname{tr} H_{k(n)}^{2}\right)^{2} .
\end{aligned}
$$


Applying Lemma 1 to (3.5), we get

$$
\begin{aligned}
\sum_{\alpha \beta} \operatorname{tr}\left(H_{\alpha} H_{\beta}-H_{\beta} H_{\alpha}\right)^{2}-\sum_{\alpha \beta}\left(\operatorname{tr} H_{\alpha} H_{\beta}\right)^{2} \geq-\frac{3}{2}\left(\sum_{\alpha \neq k(n)} \operatorname{tr} H_{\alpha}^{2}\right)^{2}-\left(\operatorname{tr} H_{k(n)}^{2}\right)^{2} \\
=-\frac{3}{2}\left(S-\operatorname{tr} H_{k(n)}^{2}\right)^{2}-\left(\operatorname{tr} H_{k(n)}^{2}\right)^{2}=-\frac{3}{2}\left(S-n H^{2}\right)^{2}-n^{2} H^{4}=-\frac{3}{2} S^{2}+3 n H^{2} S-\frac{5}{2} n^{2} H^{4} .
\end{aligned}
$$

On the other hand, by (3.1) we have

$$
\sum_{i j k \alpha}\left(h_{i j k}^{\alpha}\right)^{2} \geq \sum_{i k}\left(h_{i i k}^{k(n)}\right)^{2}=n \sum_{i}\left(\nabla_{i} H\right)^{2}=n|\nabla H|^{2} .
$$

It is obvious that

$$
\frac{1}{2} \Delta H^{2}=H \Delta H+|\nabla H|^{2} .
$$

Therefore, using Lemma 2, (2.6) and (2.7) by (3.4) we get

$$
\begin{aligned}
\frac{1}{2} \Delta S= & \sum_{i j k \alpha \alpha}\left(h_{i j k}^{\alpha}\right)^{2}+\sum_{i j \alpha} h_{i j}^{\alpha} \Delta h_{i j}^{\alpha} \\
= & \sum_{i j k \alpha}\left(h_{i j k}^{\alpha}\right)^{2}+n H \Delta H+\frac{c}{4}(n+1) S+n H^{2} S-n^{2} H^{2} c \\
& +\sum_{\alpha \beta} \operatorname{tr}\left(H_{\alpha} H_{\beta}-H_{\beta} H_{\alpha}\right)^{2}-\sum_{\alpha \beta}\left(\operatorname{tr} H_{\alpha} H_{\beta}\right)^{2} \\
\geq & \sum_{i j k \alpha}\left(h_{i j k}^{\alpha}\right)^{2}+n H \Delta H+\frac{c}{4}(n+1) S+4 n H^{2} S-n^{2} H^{2} c-\frac{3}{2} S^{2}-\frac{5}{2} n^{2} H^{4} \\
\geq & n|\nabla H|^{2}+n H \Delta H+\frac{c}{4}(n+1) S+4 n H^{2} S-n^{2} H^{2} c-\frac{3}{2} S^{2}-\frac{5}{2} n^{2} H^{4} \\
= & \frac{1}{2} n \Delta H^{2}+\frac{c}{4}(n+1) S+4 n H^{2} S-\frac{3}{2} S^{2}-n^{2} H^{2} c-\frac{5}{2} n^{2} H^{4} .
\end{aligned}
$$

Since $N$ is compact, we obtain from (3.8)

$$
\int_{N}\left\{6 S^{2}-\left[(n+1) c+16 n H^{2}\right] S+4 n^{2} H^{2} c+10 n^{2} H^{4}\right\} d N \geq 0 .
$$

From the first inequality of (3.8) we know that if $N$ is compact and

$$
6 S^{2}-\left[(n+1) c+16 n H^{2}\right] S+4 n^{2} H^{2} c+10 n^{2} H^{4}-4 n H \Delta H \leq 0,
$$

then $\sum_{i j k \alpha}\left(h_{i j k}^{\alpha}\right)^{2}=0$, that is, the second fundamental form $h_{i j}^{\alpha}$ is parallel. In particular, when the equality of (3.9) holds, we see from (3.8) that the equality

$$
\sum_{\alpha \beta \neq k(n)} \operatorname{tr}\left(H_{\alpha} H_{\beta}-H_{\beta} H_{\alpha \gamma}\right)^{2}-\sum_{\alpha \beta \neq k(n)}\left(\operatorname{tr} H_{\alpha} H_{\beta}\right)^{2}=-\frac{3}{2}\left(\sum_{\alpha \neq k(n)} \operatorname{tr} H_{\alpha \gamma}^{2}\right)^{2}
$$


holds. Thus, by Lemma 1 we see that (i) $H_{\alpha}=0(\alpha \neq k(n))$ or (ii) there exist two non-zero $H_{\alpha}$. In the case (i), we get $S=n H^{2}$. Hence noting $H=$ constant and substituting it into the equality of (3.9), we obtain

$$
(3 n-1) c n H^{2}=0 .
$$

This implies $H=0$, so that $N$ is totally geodesic or $c=0$ so that $N$ is flat. Now, we will prove that the case (ii) can not occur. Otherwise, using the same method as in [3]), we may see $n=2$. Thus we may assume

$$
H_{l(1)}=\left(\begin{array}{cc}
a & 0 \\
0 & -a
\end{array}\right), \quad H_{l(2)}=\left(\begin{array}{cc}
0 & a \\
a & 0
\end{array}\right), \quad H_{K(2)}=\left(\begin{array}{cc}
H & 0 \\
0 & H
\end{array}\right), \quad H_{\alpha}=0 .
$$

Here $a \neq 0, \alpha \neq I(1), I(2), K(2)$.

Let the codimension of $N$ be $p(=3 n)$. Put

$$
\begin{gathered}
S_{\alpha}=\sum_{i j}\left(h_{i j}^{\alpha}\right)^{2}, \\
p \sigma_{1}=\sum_{\alpha} S_{\alpha}=S, \\
p(p-1) \sigma_{2}=2 \sum_{\alpha<\beta} S_{\alpha} S_{\beta} .
\end{gathered}
$$

It can be easily seen (cf. [3])

$$
p^{2}(p-1)\left(\sigma_{1}^{2}-\sigma_{2}\right)=\sum_{\alpha<\beta}\left(S_{\alpha}-S_{\beta}\right)^{2} .
$$

By a direct calculation using (3.10), we get

and

$$
\begin{gathered}
p^{2}(p-1) \sigma_{1}^{2}=(p-1)\left(4 a^{2}+2 H^{2}\right)^{2}, \\
p^{2}(p-1) \sigma_{2}=p\left(8 a^{4}+16 a^{2} H^{2}\right),
\end{gathered}
$$

$$
\sum_{\alpha<\beta}\left(S_{\alpha}-S_{\beta}\right)^{2}=8\left(a^{2}-H^{2}\right)^{2}
$$

Substituting (3.12)-(3.14) into (3.11), we obtain

$$
(p-1)\left(4 a^{2}+2 H^{2}\right)^{2}-p\left(8 a^{4}+16 a^{2} H^{2}\right)=8\left(a^{2}-H^{2}\right)^{2} .
$$

From (3.15) we get

$$
(p-3)\left(2 a^{4}+H^{4}\right)=0,
$$

namely

$$
(3 n-3)\left(2 a^{4}+H^{4}\right)=0,
$$

implying $n=1$, because $2 a^{4}+H^{4} \neq 0$. This is a contradiction, since $n=2$. 


\section{REFERENCES}

1. B. Y. Chen and C. S. Houh, Totally real submanifolds of a quaternion projective space, Ann. di Math. Pura Appl. 120 (1979), 185-199.

2. B. Y. Chen, Some results of Chern-do Carmo-Kobayashi type and the length of second fundamental form, Indiana Univ. Math. J. 20 (1971), 1175-1185.

3. S. S. Chern, M. do Carmo and S. Kobayashi, Minimal submanifolds of a sphere with second fundamental form of constant length, in Functional Analysis and Related Field (Springer-Verlag, New York, 1970) p. 60-75.

4. A. M. Li and J. M. Li, An intrinsic rigidity theorem for minimal submanifolds in a sphere, Arch. Math. 58 (1992), 582-594.

Department of Mathematics

TOKYO METROPOLITAN UNIVERSITY

HACHIOJI, TOKYO, 192-03

JAPAN 